\title{
Efficient Space/Time Compression to Reduce Test Data Volume and Testing Time for IP Cores ${ }^{\star}$
}

\author{
Lei $\mathrm{Li}^{1}$, Krishnendu Chakrabarty ${ }^{1}$, Seiji Kajihara ${ }^{2}$ and Shivakumar Swaminathan ${ }^{3}$ \\ ${ }^{1}$ Dept. Electrical \& Computer Engineering \\ ${ }^{2}$ Dept. Computer Sciences \& Electronics \\ ${ }^{3}$ IBM Microelectronics \\ Duke University, Durham, NC 27708 \\ Kyushu Institute of Technology, Japan \\ E-mail: $\{11, k r i s h\} @ e e . d u k e . e d u$ \\ E-mail: kajihara@cse.kyutech.ac.jp \\ Research Triangle Park, NC \\ E-mail: s9s@us.ibm.com
}

\begin{abstract}
We present two-dimensional (space/time) compression techniques that reduce test data volume and test application time for scan testing of intellectual property (IP) cores. We start with a set of test cubes and use the well-known concept of scan chain compatibility to determine a small number $c$ of tester channels that are needed to drive $m$ scan chains $(c \ll m)$. Next, we exploit logic dependencies between the test data for the scan chains to design a single-level decompression circuit based on two-input gates. We refer to these procedures collectively as width (space) compression. We then determine a small set of test patterns that can provide complete fault coverage when they are applied to the circuit under test using the $c$ tester channels; this procedure is referred to as height (time) compression. In this way, structural information about the IP cores is not necessary for fault simulation, dynamic compaction, or test generation. The hardware overhead of the proposed approach is limited to the fan-out structure and a very small number of gates between the tester-driven external scan pins and the internal scan chains. Results are presented for the ISCAS-89 benchmarks and for four industrial circuits.
\end{abstract}

\section{INTRODUCTION}

Recent advances in process technology have led to a rapid increase in the density of integrated circuits (ICs). The increased density and the need to test for new types of defects in nanometer technologies have resulted in a tremendous increase in test data volume and test application time. The test data volume for ICs in 2014 is projected to be as much as 150 times the test data volume in 1999 [10].

In addition to the increasing density of ICs, today's systemon-chip (SOC) designs also exacerbate the test data volume problem. The increase in test data volume not only leads to the increase of testing time, but the high test data volume may also exceed the limited memory depth of automatic test equipment (ATE). Multiple ATE reloads are time-consuming since data transfer from a workstation to the ATE hard disk or from the ATE hard disk to ATE channels are relatively slow; the upload time ranges from tens of minutes to hours [17]. While test application time for scan vectors can be reduced by using a large number of internal scan chains, the number of internal scan chains that can be driven by an ATE is limited in practice due to pin count constraints.

Test data volume for IP cores can be reduced by compressing the precomputed test set $T_{D}$ provided by the core vendor to a much smaller data set $T_{E}$, which is stored in ATE memory. An on-chip decoder is used for pattern decompression to generate

\footnotetext{
^ This research was supported in part by the National Science Foundation under grants CCR-9875324, CCR-0204077, and OISE-0403217, and by a graduate fellowship from the IEEE/ACM Design Automation Conference.
}

$T_{D}$ from $T_{E}$ during pattern application $[3,5,19]$. Such techniques are typically based on run-length codes and their variants, e.g., frequency-directed run-length (FDR) codes. However, most methods based on compression codes target single scan chains and they require complex synchronization between the ATE and the circuit under test. Test data volume reduction techniques based on on-chip linear decompression hardware [11, 14] and hybrid BIST [9] have also been presented in the literature. However, these techniques utilize structural information about the circuit under test, which prevents their applicability to IP cores.

In this paper, we present two-dimensional (space/time) compression techniques that reduce test data volume and testing time by allowing a large number of internal scan chains to be driven by a small number of tester channels. We start with a set of precomputed test cubes and use the well-known concept of scan chain compatibility to determine the number $c$ of tester channels that are needed to drive $m$ scan chains $(c \ll m)$. Next, as sketched in Figure 1, we use the logic dependencies between the test data for the scan chains to design a decompression logic based on a single level of two-input gates. We refer to these procedures collectively as width (space) compression. We then determine a small set of test patterns that can provide complete fault coverage when they are applied to the circuit under test using the $c$ tester channels; this procedure is referred to as height (time) compression. This approach does not require structural details of the IP core for fault simulation or test generation. It facilitates test pattern reuse because no additional test generation or fault simulation are required during system integration.

A number of related techniques for two-dimensional compression based on test cubes rely on the interleaving of the width and height compression procedures [1]. Such methods require tailored test development solutions that can support incremented test generation as the compression circuit is determined; thus even though these methods are non-intrusive in term of circuit redesign, they require structural information about the circuit under test. In contrast, height compression in this paper is carried out after width compression is accomplished, and without the use of any test generation. There is no loss in fault coverage because all the test patterns in $T_{D}$ are applied to the circuit under test.

The steady increase in clock frequencies over the recent past has led to designs with a small number of gates between latches, or between latches and I/O pins. As a result, current-generation logic circuits have very short combinational logic depth, and a 
large number of logic cones with very little overlap. This is in contrast to older circuits such as the ISCAS-85 benchmarks that tend to have a smaller number of overlapping logic cones. A consequence of the shallow logic depth is that test patterns in present-day circuits contain many don't-care bits. A commercial test pattern generator typically uses random fill to increase the likelihood of surreptitious fault detection. However, if Xfill is used during test generation and the test sets for the cores are delivered with the don't-care bits to the system integrator, an appropriate compression method can be used at the system level to reduce test data volume and testing time. This imposes no additional burden on the core vendor.

Experimental results for the ISCAS-89 circuits demonstrate that despite the inherent simplicity and low hardware overhead, the proposed technique provide significant improvement over other recent methods. We also present results for four production circuits from IBM, and show that compared to the methods employed for production testing of these circuits, the proposed technique leads to $10 \mathrm{X}$ reduction over compacted test sets in both test data volume and testing time.

The rest of the paper is organized as follows. In Section II, we present the proposed compression techniques. Section III discuss the impact of the number of ATE channels on the test application time. Experimental results and a comparison with related recent work for IP cores are presented in Section IV. Finally, Section V concludes the paper.

\section{PRoposed ApPROACH}

The proposed two-dimensional test data compression techniques consist of two steps, namely width compression and height compression. Consider a test set of $p$ test cubes for $m$ internal scan chains. The "width" of the test data is the number of internal scan chains $m$, the "height" of a test pattern is the scan chain length $l$, and the "height" of the test set is the product of $l$ and the number of test patterns $p$. Here we assume that the scan chains are balanced. For unbalanced scan chains, don't-care bits can be used to pad the shorter scan chains. The first step in our approach is referred to as width compression, in which we use $c$ ATE channels to drive the $m$ scan chains, where $c \ll m$. Width compression is achieved by exploiting the compatibilities and the logic dependencies between the scan chains for the precomputed test cubes. In the second step, we determine a set of test patterns that can detect all the irredundant faults in the circuit; the test data is compressed now in the height dimension. Height compression is achieved through static compaction on the set of precomputed test cubes.

\section{A. Width Compression}

In the width compression step, $c$ ATE channels are used to drive $m$ scan chains, where $c$ is much less than $m$. In this way, the test patterns are compressed in the width dimension. The parameter $c$ is determined based on the compatibilities and the logic dependencies between the scan chains for different test cubes. The concept of scan chain compatibility and the associated fan-out structure have been utilized in a number of papers $[1,7]$ since the broadcast scan architecture was presented

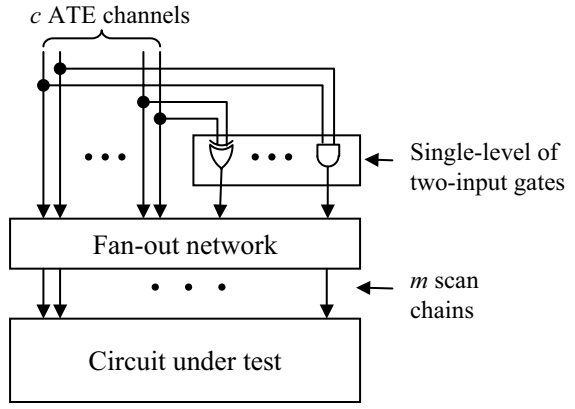

Fig. 1. Fan-out structure.

in [12]. In addition to the compatibilities between the scan chains, we also exploit the logic dependencies between the scan chains to reduce $c$.

Let $b_{k, i, j}$ denote the data bit that is shifted into the $j$ th flipflop of $i$ th scan chain for the $k$ th test pattern. The compatibility of a pair of scan chains is defined as follows. The $i_{1}$ th scan chain and the $i_{2}$ th scan chain are mutually compatible if for any $k(1 \leq k \leq p)$ and $j(1 \leq j \leq l), b_{k, i_{1}, j}$ and $b_{k, i_{2}, j}$ are either equal to each other or at least one of them is a don't-care. We next form a conflict graph from the test data and use graph coloring solution to obtain the number of ATE channels needed. The conflict graph $G$ contains a vertex for each scan chain. If two scan chains are not compatible, they are connected by an edge in $G$. A vertex coloring of $G$ yields the minimum number of ATE channels required to apply the test cubes with no loss of fault coverage (In the graph coloring solution, a minimum number of colors is determined to color the vertices such that no two adjacent vertices are assigned the same color.) Vertices in $G$ that are assigned the same color represent scan chains that can be fed by the same ATE channel via a fan-out structure. This procedure is illustrated using Figures 2.

In the above example, the number of scan chains $m=8$, and the scan chain length $l=4$. A conflict graph consisting 8 vertices is first determined. The coloring solution for the conflict graph is also shown; three colors are used to color three sets of vertices: $\{2,4,5,7\},\{3,6,8\}$ and $\{1\}$. Consequently, three ATE channels are needed to feed the test data for the 8 scan chains, and the fan-out structure is shown in the figure. Since the graph coloring problem is known to be NP-complete, we use the simple heuristic described in [13].

While a significant amount of space compression can be achieved by exploiting the compatibilities between the scan chains and using a fan-out structure with $c^{\prime}$ inputs to drive $m$ internal scan chains, we can achieve additional space compression by investigating the logic dependencies between the $c^{\prime}$ data streams from the ATE. In this way, the $c^{\prime}$ channels from the ATE can be reduced to $c<c^{\prime}$ channels. The following discussion generalizes the design technique introduced in [8] for BIST of combinational logic circuits. Let $b_{k, i, j}$ denote the $j$ th data bit that is shifted to the $i$ th input of the fan-out structure for the $k$ th test pattern. The $i$ th input is logically dependent on a set of inputs $\left\{u_{1}, u_{2}, \ldots, u_{s}\right\}$ if there exists an $s$-input logic function $F$ such that for any $k(1 \leq k \leq p)$ and $j(1 \leq j \leq l)$, 


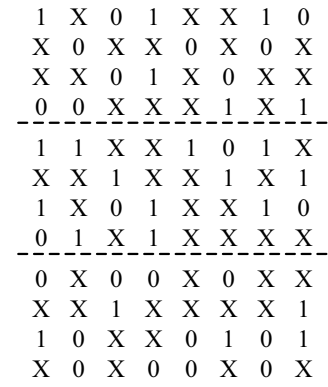

(a) Test cubes

\begin{tabular}{ccc}
1 & 1 & 0 \\
$\mathrm{X}$ & 0 & $\mathrm{X}$ \\
$\mathrm{X}$ & 1 & 0 \\
0 & $\mathrm{X}$ & 1 \\
\hdashline 1 & 1 & 0 \\
$\mathrm{X}$ & $\mathrm{X}$ & 1 \\
1 & 1 & 0 \\
0 & 1 & $\mathrm{X}$ \\
\hline 0 & 0 & 0 \\
$\mathrm{X}$ & $\mathrm{X}$ & 1 \\
1 & 0 & 1 \\
$\mathrm{X}$ & 0 & $\mathrm{X}$
\end{tabular}

(c) Compressed test data based on scan chain compatibility

Fig. 2. Width compression based on scan chain compatibilities.

$b_{k, i, j}=F\left(b_{k, u_{1}, j}, b_{k, u_{2}, j}, \ldots, b_{k, u_{s}, j}\right)$. The don't-care bits among $b_{k, i, j}, b_{k, u_{1}, j}, b_{k, u_{2}, j}, \ldots, b_{k, u_{s}, j}$ can be set appropriately to satisfy the above equation. If the $i$ th input is logically dependent on an input set $\left\{u_{1}, u_{2}, \ldots, u_{s}\right\}$, then a $s$-to- 1 combinational circuit can be used to generate the test data for the $i$ th input from the inputs $\left\{u_{1}, u_{2}, \ldots, u_{s}\right\}$ and the number $c$ of ATE channels needed to drive the fan-out structure is reduced by one. For $c$ inputs, the complexity for checking all possible logic dependencies with a specific $s$-input logic function is $O\left(p l c\left(\begin{array}{l}c \\ s\end{array}\right)\right)$. Since there are a total of $2^{2^{s}}$ different $s$-input logic functions, the complexity for checking all the possible logic dependencies with any $s$-input logic function is $O\left(p l c\left(\begin{array}{l}c \\ s\end{array}\right) 2^{2^{s}}\right)$. While in many cases, the logic dependency check can terminate before going though all the $p l$ bits, the computational complexity is still prohibitive for large $s$. In this paper, we only examine the logic dependencies for the six 2-input functions: AND, NAND, OR, NOR, XOR, and XNOR. This restriction also reduces the hardware overhead required to generate the data for an input channel from the data for other input channels.

Consider two $v$-bit data streams $X=x_{1}, x_{2}, \ldots, x_{v}$ and $Y=y_{1}, y_{2}, \ldots, y_{v}$, respectively, where $x_{i}, y_{i} \in\{0,1, x\}, 1 \leq$ $i \leq v$. Suppose that the two data streams are combined using a two-input gate, denoted by $\otimes$, to generate a third data stream $Z=z_{1}, z_{2}, \ldots, z_{v}$, where $z_{i}=x_{i} \otimes y_{i}, 1 \leq i \leq v$. Table I shows the necessary and sufficient conditions under which gate type $\otimes$ can be used to generate $z_{i}$ from $x_{i}$ and $y_{i}, 1 \leq i \leq v$, for three different gate types. Note that $Z$ can be generated from $X$ and $Y$ using gate type $\otimes$ if and only if $z_{i}$ can be generated from $x_{i}$ and $y_{i}$ for all $i, 1 \leq i \leq v$.

Let $\mathcal{G}$ be the set of available two-input gates, i.e.,
TABLE I. NECESSARY AND SUFFICIENT CONDITIONS TO GENERATE $z_{i}$ FROM $x_{i}$ AND $y_{i}$ USING GATE TYPE $\bigotimes$.

\begin{tabular}{|c|c|c|c|c|c|c|c|}
\hline$\otimes$ & $z_{i}$ & $x_{i}$ & $y_{i}$ & $\otimes$ & $z_{i}$ & $x_{i}$ & \\
\hline \multirow{3}{*}{ AND } & 0 & $\begin{array}{l}0 \\
X \\
X\end{array}$ & $\begin{array}{l}\mathrm{X}^{1} \\
0 \\
\mathrm{X}(\text { map to } 0)\end{array}$ & \multirow{6}{*}{ XOR } & \multirow[b]{2}{*}{0} & \multirow{2}{*}{$\begin{array}{l}0 \\
0 \\
\mathrm{X} \text { (map to } 0) \\
1 \\
1 \\
\mathrm{X} \text { (map to } 1) \\
\mathrm{X}(\text { map to } 0)\end{array}$} & \multirow{2}{*}{$\begin{array}{l} \\
X \text { (map to } 0) \\
0 \\
1 \\
X(\text { map to } 1) \\
1 \\
X \text { (map to } 0)\end{array}$} \\
\hline & 1 & $\begin{array}{l}1 \\
1 \\
X \text { (map to } 1) \\
X(\text { map to } 1)\end{array}$ & $\begin{array}{l}1 \\
X \text { (map to } 1) \\
1 \\
X(\text { map to } 1)\end{array}$ & & & & \\
\hline & $X$ & $\mathrm{X}$ & $\mathrm{X}$ & & \multirow{3}{*}{1} & & \\
\hline \multirow[t]{4}{*}{ OR } & 0 & $\begin{array}{l}0 \\
0 \\
X(\text { map to } 0) \\
X(\text { map to } 0)\end{array}$ & $\begin{array}{l}0 \\
X(\text { map to } 0) \\
0 \\
X(\text { map to } 0)\end{array}$ & & & $\begin{array}{l}0 \\
\mathrm{X} \text { (map to } 1) \\
1 \\
1\end{array}$ & $\begin{array}{l}X \text { (map to } 1) \\
0 \\
0 \\
X(\text { map to } 0)\end{array}$ \\
\hline & \multirow[t]{2}{*}{1} & $\begin{array}{l}1 \\
\mathrm{X}\end{array}$ & $\begin{array}{l}\mathrm{X} \\
1\end{array}$ & & & $\begin{array}{l}X \text { (map to } 0) \\
X \text { (map to } 0)\end{array}$ & $\begin{array}{l}1 \\
X(\text { map to } 1)\end{array}$ \\
\hline & & $\mathrm{X}$ & $\mathrm{X}$ (map to 1$)$ & & $\mathrm{X}$ & $\mathrm{X}$ & $\mathrm{X}$ \\
\hline & $\mathrm{X}$ & $\mathrm{X}$ & $\mathrm{X}$ & & & & \\
\hline
\end{tabular}

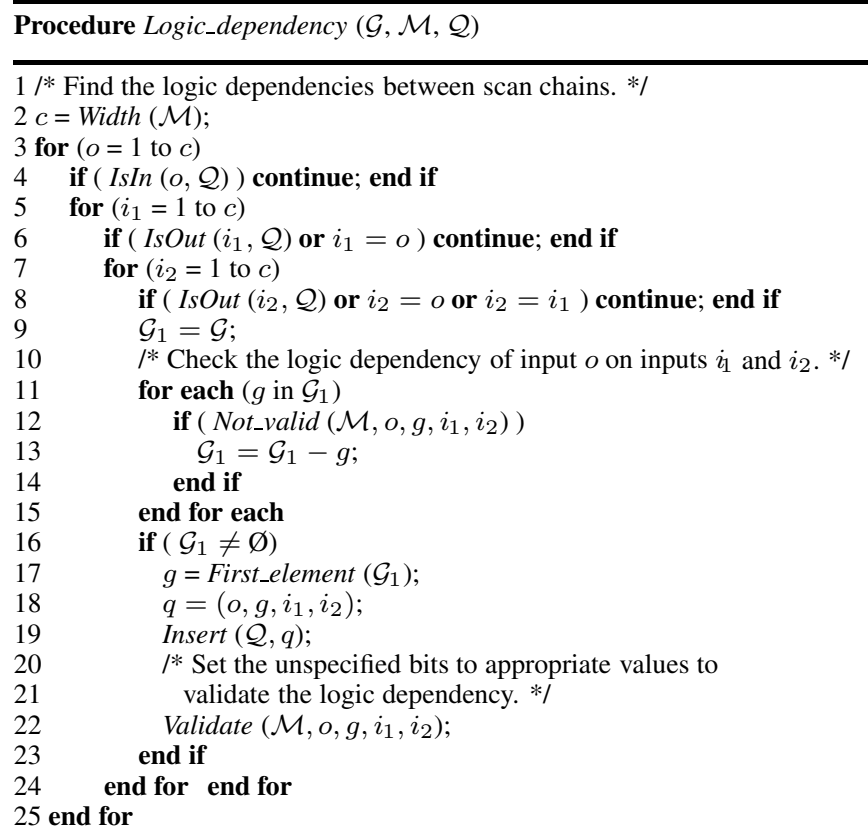

Fig. 3. Pseudocode determine the logic dependencies between scan chains.

$\mathcal{G}=\{$ AND, NAND, OR, NOR, XOR, XNOR $\}$, and $\mathcal{M}$ be the compressed test data in matrix form (with $c^{\prime}$ columns and $p l$ rows) after exploiting the compatibilities between scan chains. The procedure used in our experiments to find the logic dependencies between scan chains is described in Figure 3. The output of the procedure is a set $\mathcal{Q}$ in which each element is a 4-tuple $\left(o, g, i_{1}, i_{2}\right)$. The 4-tuple $\left(o, g, i_{1}, i_{2}\right)$ indicates that the data for the input channel $o$ can be generated from the data for the input channels $i_{1}$ and $i_{2}$ using a two-input gate type $g$. Some of the don't-care bits in the compressed test data matrix $\mathcal{M}$ are set to appropriate values in accordance with logic dependencies identified by the procedure. The function $\operatorname{Is} \operatorname{In}(\mathcal{Q}, i)$ is used to find out if the input channel $i$ is the input of any of the 4-tuples in $\mathcal{Q}$. Similarly, the function $\operatorname{Is} \operatorname{Out}(\mathcal{Q}, i)$ is used to find out if the input channel $i$ is the output of any of the 4-tuples in $\mathcal{Q}$. Applying the procedure to the compressed test data in Figure 2(c), we can obtain the output $\mathcal{Q}=\{(1, \mathrm{XOR}, 2,3)\}$. The resulting compressed test data and gated fan-out structure are shown in Figure 4. 
As a result of the width compression procedure, constraints are introduced for the test patterns, i.e., the scan chains fed by the same ATE channel are filled with the same test data for any test pattern. However, as long as all the irredundant faults can be detected by the original test set that is used to obtain the fan-out structure, they can also be detected by the test patterns arising from the constraints that are introduced by the fan-out structure. It is easy to show that if a fault is detectable in the original circuit with $m$ external channels, it is also detectable in the circuit with $c$ external channels obtained after width compression. In contrast to [1], no attempt is made here to make $c$ any smaller by relaxing care bits or by interleaving width compression with test generation.

\section{B. Height Compression}

In this subsection, we describe the procedure for height compression. Assuming that structural information about the IP core is not available or test reuse if preferred (test generation is deemed to be too expensive), we first merge the test data of the compatible scan chains for the original test set after the width compression procedure. Next, static compression is carried out with the width-compressed test data to reduce the number of test patterns. The fault coverage achieved with the fanout structure and with the test patterns obtained after static compaction is the same as the fault coverage obtained with the original set of $p$ test cubes and $m$ external channels. The static compaction algorithm is similar to the width compression of the previous subsection in that it also relies on graph coloring. The difference lies in that fact that each vertex corresponds to a test pattern instead of a scan chain. This approach does not require any test generation or fault simulation, hence it is especially attractive for hard cores in SOCs.

\section{IMPACT OF THE NUMBER OF ATE CHANNELS}

In conventional testing using multiple scan chains, the number of ATE channels is equal to the number of scan chains. An increase in the number of ATE channels leads to a reduction in testing time because more data can be shifted into the scan chains in a single scan clock cycle. However, in the fan-out approach, the number of internal scan chains $m$ can be very large, even for a small number of ATE channels. In practice, it may not be possible to increase $m$ beyond a certain limit due to routing constraints. In such situations, an increase in the number of ATE channels only provides the benefit of more freedom for height compression; the number of data bits that are shifted into the scan chains in a single cycle is not increased. Thus there is only limited benefit in increasing the number of ATE channels any further. This issue is highlighted in the experimental results obtained for two production circuits from IBM, namely CKT1 and CKT2.

CKT1 consists of 51082 gates and 10788 latches and its test set provides $99.80 \%$ fault coverage. CKT2 consists of 94340 gates and 17915 latches and its test set provides $99.76 \%$ fault coverage. The detailed experimental results for these two circuits are listed in Section IV. Here we only show the impact of the number of ATE channels on the test application time. We

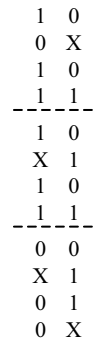

(a) Compressed test data based on the compatibilities and logic dependencies

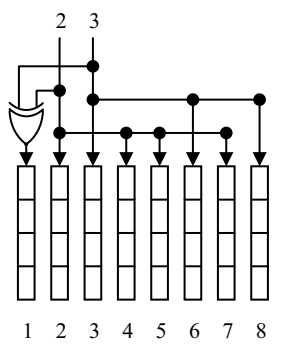

(b) Gated fan-out structure
Fig. 4. Width compression based on scan chain compatibilities and logic dependencies.

limit the number of scan chains to 600 for CKT1 and 1000 for CKT2, respectively. For CKT1, we vary the number of scan chains from 128 to 600, and apply the width compression procedure to determine the minimum number of ATE channels that are needed to drive the scan chains. For 600 scan chains, we find that the minimum number of ATE channels needed is 32 . Next, we fix the number of scan chains at 600 while continuing to increase the number of ATE channels. The relationship of the number of internal scan chains to the number of ATE channels is shown in Figure 5(a). For each pair of $(c, m)$ values shown in Figure 5(a) for CKT1, we perform static compaction to reduce the number of test patterns, and derive the test application time. Figure 5(b) shows the testing time versus the number of ATE channels. After the number of ATE channels reaches 32, the increase in the number of ATE channels does not lead to significant reduction of testing time for the proposed method. In Figure 5(c), we also show the testing time for the ATPG-compacted test set versus the number of ATE channels. The figure shows that even though the testing time for the latter case continues to decrease with an increase in $c$, it is only for very large values of $c$ that the testing time for this case becomes comparable to that for the proposed methods. Similar results are obtained for CKT2, as shown in Figure 5.

\section{EXPERIMENTAL RESULTS}

In this section, we first apply the proposed approach to the seven largest ISCAS-89 benchmark circuits. For each circuit, we start with a test set obtained from the Mintest ATPG program [6] without dynamic compaction. We first use the width compression technique described in Section II-A to obtain the minimum number of ATE channels that are needed to drive the scan chains, and we determine the corresponding fan-out structure. Since these test sets provide $100 \%$ fault coverage for the irredundant single stuck-at faults, the width compression procedure guarantees that all the irredundant faults can be detected by test data transferred through the fan-out structure. After width compression, we apply height compression techniques to reduce the number of test patterns. Table II shows the results for height compression based on static compaction. Static compaction took less then 70 seconds for each of the ISCAS-89 circuits.

In Table II, we also compare the test data volume and testing 


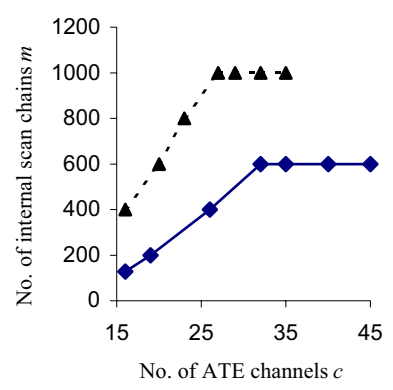

(a)

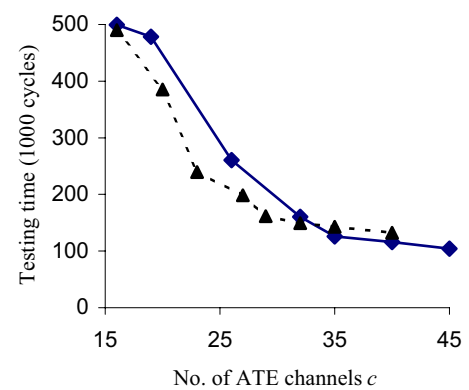

(b)

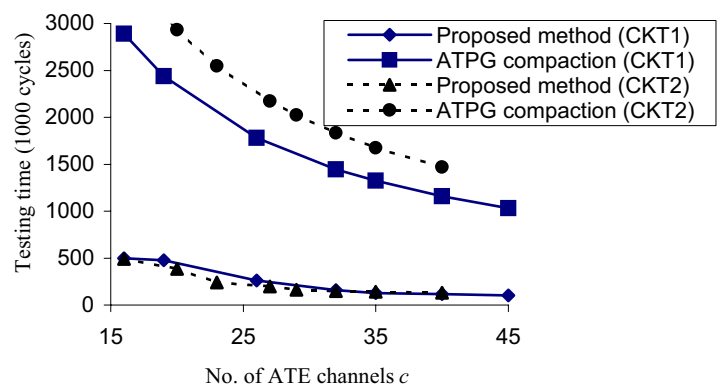

(c)

Fig. 5. The impact of the number of ATE channels on testing time for CKT1 and CKT2.

TABLE II. COMPRESSION RESULTS FOR THE ISCAS-89 BENCHMARK CIRCUITS.

\begin{tabular}{|c|c|c|c|c|c|c|c|c|c|c|c|c|c|c|c|}
\hline Circuit & $\begin{array}{l}\text { No. of } \\
\text { test } \\
\text { cubes }\end{array}$ & $\begin{array}{l}\text { No. of } \\
\text { scan } \\
\text { chains }\end{array}$ & $\begin{array}{c}\text { No. of } \\
\text { ATE } \\
\text { channels }\end{array}$ & $\begin{array}{c}\text { No. of } \\
\text { two-input } \\
\text { gates } \\
\text { required }\end{array}$ & $\begin{array}{l}\text { No. of } \\
\text { patterns } \\
\text { for } T_{E}\end{array}$ & $\begin{array}{l}\left|T_{E}\right| \\
\text { (bits) }\end{array}$ & $\begin{array}{l}T A T_{E} \\
\text { (cycles) }\end{array}$ & $\begin{array}{l}\left|T_{\mathrm{M}}\right| \\
\text { (bits) }\end{array}$ & $\begin{array}{l}T A T_{\mathrm{M}} \\
\text { (cycles) }\end{array}$ & $\begin{array}{l}\triangle V(\%) \\
\text { (Mintest) }\end{array}$ & $\begin{array}{c}\triangle T A T(\%) \\
\text { (Mintest) }\end{array}$ & $\begin{array}{l}\left|T_{\mathrm{A}}\right| \\
\text { (bits) }\end{array}$ & $\begin{array}{l}T A T_{\mathrm{A}} \\
\text { (cycles) }\end{array}$ & $\begin{array}{l}\triangle V(\%) \\
\text { (Atalanta) }\end{array}$ & $\begin{array}{c}\triangle T A T(\%) \\
\text { (Atalanta) }\end{array}$ \\
\hline s5378 & 1458 & 64 & 9 & 4 & 395 & 14220 & 1975 & 23754 & 2775 & 40.14 & 28.83 & 52858 & 6175 & 73.10 & 68.02 \\
\hline s9234 & 1928 & 64 & 16 & 7 & 471 & 30144 & 2355 & 39273 & 2703 & 23.24 & 12.87 & 88426 & 6086 & 65.91 & 61.30 \\
\hline s13207 & 3237 & 200 & 11 & 4 & 477 & 20988 & 2385 & 165200 & 15340 & 87.30 & 84.45 & 323400 & 30030 & 93.51 & 92.06 \\
\hline \multirow[b]{2}{*}{ s15850 } & 3920 & 200 & 15 & 2 & 422 & 25320 & 2110 & 76986 & 5292 & 67.11 & 60.13 & 265785 & 18270 & 90.47 & 88.45 \\
\hline & 3920 & 128 & 12 & 2 & 419 & 25140 & 2514 & 76986 & 6552 & 67.34 & 61.63 & 265785 & 22620 & 90.54 & 88.89 \\
\hline s35932 & 10810 & 200 & 3 & 1 & 147 & 3969 & 1470 & 28208 & 9424 & 85.93 & 84.40 & 111069 & 37107 & 96.43 & 96.04 \\
\hline \multirow[b]{2}{*}{ s38417 } & 10771 & 200 & 14 & 0 & 1129 & 142254 & 11290 & 164736 & 11880 & 13.65 & 4.97 & 1560832 & 112560 & 90.89 & 89.97 \\
\hline & 10771 & 48 & 5 & 0 & 487 & 85225 & 17532 & 164736 & 33066 & 48.27 & 46.98 & 1560832 & 313292 & 94.54 & 94.40 \\
\hline s38584 & 13468 & 200 & 14 & 2 & 510 & 57120 & 4590 & 199104 & 14416 & 71.31 & 68.16 & 900360 & 65190 & 93.66 & 92.96 \\
\hline
\end{tabular}

time to the case when a set of ATPG-compacted test patterns $T_{\mathrm{M}}\left(T_{\mathrm{A}}\right)$ obtained using Mintest (Atalanta) is applied through the same number of external ATE channels. The parameters $\triangle V$ and $\triangle T A T$ refer to the reduction in test data volume and and test application time, respectively, over ATPG compaction. While we achieve lower test data volume and testing time in all cases with negligible hardware overhead, the reduction is especially striking for s13207 and s35932.

In Table III, we compare our results to some recentlypublished test data compression methods that do not utilize structural information of the circuit under test. We report the smallest test data volume obtained over several values of $m$. Compared to FDR and 9C (EFDR and VIHC), the test data volume of our proposed approach is higher in three (four) out of the seven cases. The proposed method outperforms LZ77 in five out seven cases. We are unable to directly compare our results with [19] because of the different test sets used in [19]. Note however that coding methods such as $[3,4,5,19]$ are targeted towards single scan chains; for multiple scan chains, these methods either require considerable hardware overhead (decoder per scan chain) or higher testing time. In 9C coding [16], an $m$-bit shifter is required for the decompression architecture if it drives $m$ scan chains. In [18], the boundary scan cells need to be modified and used for decompression. Unlike FDR [3], EFDR [4], VIHC [5] and 9C coding [16], the proposed method does not require synchronization with the ATE.

In order to demonstrate the effectiveness of the proposed two-dimensional test data compression techniques for industrial circuits, we present results for four production circuits from IBM. CKT1 and CKT 2 have been described in Section III. CKT3 consists of 3.6 million gates and 726000 latches, and
TABLE III. COMPARISON OF COMPRESSION RESULTS

\begin{tabular}{|c|c|c|c|c|c|c|}
\hline \multirow{2}{*}{ Circuit } & \multicolumn{7}{|c|}{ Size of $T_{E}$ (bits) } \\
\cline { 2 - 7 } & $\begin{array}{c}\text { Proposed } \\
\text { approach }\end{array}$ & $\begin{array}{c}\text { FDR } \\
{[3]}\end{array}$ & $\begin{array}{c}\text { EFDR } \\
{[4]}\end{array}$ & $\begin{array}{c}\text { VIHC } \\
{[5]}\end{array}$ & $\begin{array}{c}\text { 9C } \\
{[16]}\end{array}$ & $\begin{array}{c}\text { LZ77 } \\
{[18]}\end{array}$ \\
\hline s5378 & 14220 & 12346 & 11419 & 11516 & 11487 & 12769 \\
\hline s9234 & 30144 & 22152 & 21250 & 17736 & 19279 & 19429 \\
\hline s13207 & 20988 & 30880 & 29992 & 27737 & 29224 & N/A \\
\hline s15850 & 25140 & 26000 & 24643 & 30271 & 25883 & 37980 \\
\hline s35932 & 3969 & 22744 & 5554 & 9458 & N/A & 22496 \\
\hline s38417 & 85225 & 93466 & 64962 & 74938 & 64857 & N/A \\
\hline s38584 & 57120 & 77812 & 73853 & 85674 & 68631 & N/A \\
\hline
\end{tabular}

CKT4 consists 1.2 million gates and 32200 latches. We were only provided with partial test sets for these circuits, thereby we are unable to report fault coverage numbers. We carry out static compaction on the test cubes after width compression for CKT1 and CKT2. For CKT3 and CKT4, the number of test patterns is small, hence we do not perform height compression for these test sets. While XOR is the predominant gate type for the smaller circuits, NAND and OR gates were also used in the decompression logic for CKT3 and CKT4. Table IV shows the test data volume and the testing time for our approach, and compares these to that obtained with the maximum compaction provided by the same ATPG tool. Compared to the ATPG-compacted test set $T_{C}$, the proposed method achieves up to $91 \%$ reduction in test data volume. With the same number of ATE channels, the test application time $T A T_{E}$ for the proposed method is over $90 \%$ less than that for the compacted test set. On a laptop with a $1.8 \mathrm{GHz} \mathrm{CPU}$ and $512 \mathrm{MB}$ memory, the computation time to obtain a complete set of results for CKT2 in Table IV is approximately 3 hours. The CPU times are less for the other circuits.

Table V compares the proposed approach with the FDR code [3] in terms of the reduction in test data volume and test 
TABLE IV. COMPRESSION RESULTS FOR THE INDUSTRIAL CIRCUITS.

\begin{tabular}{|c|c|c|c|c|c|c|c|c|c|c|c|c|c|}
\hline Circuit & $\begin{array}{l}\text { No. of } \\
\text { test } \\
\text { cubes }\end{array}$ & $\begin{array}{l}\text { No. of } \\
\text { scan } \\
\text { chains }\end{array}$ & $\begin{array}{l}\text { Scan } \\
\text { chain } \\
\text { length }\end{array}$ & $\begin{array}{l}\text { No. of } \\
\text { ATE } \\
\text { channels }\end{array}$ & $\begin{array}{l}\text { No. of } \\
\text { two-input } \\
\text { gates } \\
\text { required }\end{array}$ & $\begin{array}{c}\text { No. of } \\
\text { test } \\
\text { patterns } \\
\text { for } T_{E}\end{array}$ & $\begin{array}{c}\text { Size of } \\
T_{E} \text { (bits) }\end{array}$ & $\begin{array}{l}T A T_{E} \\
\text { (cycles) }\end{array}$ & $\begin{array}{c}\text { No. of } \\
\text { test } \\
\text { vectors } \\
\text { for } T_{C}^{*}\end{array}$ & $\begin{array}{c}\text { Size of } \\
T_{C} \text { (bits) }\end{array}$ & $\begin{array}{l}T A T_{C} \\
\text { (cycles) }\end{array}$ & $\begin{array}{l}\text { Compression } \\
\text { percentage } \\
\text { relative to } T_{C}\end{array}$ & $\begin{array}{l}\text { Percentage } \\
\text { reduction } \\
\text { in } T A T\end{array}$ \\
\hline \multirow{4}{*}{ CKT1 } & \multirow{4}{*}{17176} & 128 & 96 & 16 & 8 & 4150 & 6374400 & 402550 & 3768 & 46180608 & 2890056 & 86.20 & 86.07 \\
\hline & & 200 & 62 & 19 & 12 & 7593 & 8944554 & 478359 & 3768 & 46180608 & 2437896 & 80.63 & 80.38 \\
\hline & & 400 & 31 & 26 & 17 & 8140 & 6560840 & 260480 & 3768 & 46180608 & 1782264 & 85.79 & 85.38 \\
\hline & & 600 & 21 & 32 & 15 & 7301 & 4906272 & 160622 & 3768 & 46180608 & 1446912 & 89.38 & 88.90 \\
\hline \multirow{4}{*}{ CKT2 } & \multirow{4}{*}{43079} & 400 & 56 & 16 & 5 & 8600 & 7705600 & 490200 & 2636 & 58561376 & 3664040 & 86.84 & 86.62 \\
\hline & & 600 & 38 & 20 & 4 & 9876 & 7505760 & 385164 & 2636 & 58561376 & 2931232 & 87.18 & 86.86 \\
\hline & & 800 & 28 & 23 & 2 & 8252 & 5314288 & 239308 & 2636 & 58561376 & 2549012 & 90.93 & 90.61 \\
\hline & & 1000 & 23 & 27 & 2 & 8265 & 5132565 & 198360 & 2636 & 58561376 & 2172064 & 91.24 & 90.87 \\
\hline \multirow{3}{*}{ CKT3 } & \multirow{3}{*}{32} & 600 & 605 & 50 & 41 & 32 & 968000 & 19392 & 32 & 11613472 & 232320 & 91.66 & 91.65 \\
\hline & & 800 & 454 & 58 & 34 & 32 & 842624 & 14560 & 32 & 11613472 & 200288 & 92.74 & $\overline{92.73}$ \\
\hline & & 1000 & 363 & 68 & 38 & 32 & 789888 & 11648 & 32 & 11613472 & 170848 & 93.20 & 93.18 \\
\hline \multirow{3}{*}{ CKT4 } & \multirow{3}{*}{4} & 600 & 1719 & 73 & 43 & 4 & 501948 & 6880 & 4 & 4124288 & 56504 & 87.83 & 87.82 \\
\hline & & 800 & 1289 & 86 & 51 & 4 & 443416 & 5160 & 4 & 4124288 & 47964 & 89.25 & 89.24 \\
\hline & & 1000 & 1032 & 93 & 48 & 4 & 383904 & 4132 & 4 & 4124288 & 44352 & 90.69 & 90.68 \\
\hline
\end{tabular}

TABLE V. COMPARISON WITH FDR CODE [3].

\begin{tabular}{|c|c|c|c|c|c|c|c|}
\hline \multirow[b]{3}{*}{ Circuit } & \multirow{3}{*}{$\begin{array}{c}\text { No. of } \\
\text { ATE } \\
\text { channels }\end{array}$} & \multicolumn{2}{|c|}{ Proposed approach } & \multicolumn{4}{|c|}{ FDR [3] } \\
\hline & & Size of & $\begin{array}{c}T A T_{E} \\
\text { (ATE clock }\end{array}$ & & & $\begin{array}{r}T A T \\
\text { (ATE cloc }\end{array}$ & $\begin{array}{l}F D R \\
\text { ck cycles) }\end{array}$ \\
\hline & & $T_{E}$ (bits) & cycles) & $T_{E}$ (bits) & $\alpha$ & Upper bound & Lower bound \\
\hline CKT1 & 32 & 4906272 & 160622 & 3047472 & 4 & 1739836 & 1692219 \\
\hline CKT2 & 27 & 5132565 & 198360 & 5425352 & 4 & 9062449 & 8961979 \\
\hline$\overline{\mathrm{CKT} 3}$ & 68 & 789888 & 11648 & 243998 & 4 & 46285 & 44491 \\
\hline CKT4 & 93 & 383904 & 4132 & 201490 & 4 & 13253 & 12170 \\
\hline
\end{tabular}

application time. The comparison is carried out for the four industrial circuits. Using the method described in [2], we calculate upper and lower bounds on the testing time for the FDR code. In [3], the scan clock can run at a higher frequency $f_{\text {scan }}=\alpha f_{A T E}$ than the ATE clock frequency $f_{A T E}$, where $\alpha>1$ and is normally set to the power of 2 . We calculate the upper and lower bounds for $\alpha=4$. We also assume that the compressed data is transferred from the ATE using the same number of channels for the two methods. The results show that although the test data volume obtained using the FDR code is slightly in three out of four cases, the test application time of FDR code is at least at an order of magnitude higher than that for the proposed approach. This difference is even higher for the practical case of $\alpha=1$.

\section{CONClusion}

We have presented a two-step compression technique that can reduce test data volume and test application time with negligible hardware overhead for on-chip decompression. The hardware overhead is largely limited to the fan-out between the tester-driven external scan pins and the internal scan chains. Experimental results for the ISCAS-89 circuits demonstrate that the proposed technique provides significant improvement over other recent methods. Results for four industrial circuits show that compared to compacted test sets, up to $10 \mathrm{X}$ reduction in scan test data volume and testing time can be obtained.

\section{REFERENCES}

[1] I. Bayraktaroglu and A. Orailoglu, "Decompression hardware determination for test volume and time reduction through unified test pattern compaction and compression," Proc. VLSI Test Symp., pp. 113-118, 2003.

[2] A. Chandra and K. Chakrabarty, "Test resource partitioning and reduced pin-count testing based on test data compression", Proc. DATE Conf., pp. 598-603, 2002.
[3] A. Chandra and K. Chakrabarty, "Test data compression and test resource partitioning for system-on-a-chip using frequency-directed runlength (FDR) codes," IEEE Trans. Computers, vol. 52, pp. 1076-1088, August 2003.

[4] A. El-Maleh and R. Al-Abaji, "Extended Frequency-Directed RunLength Codes with Improved Application to System-on-a-Chip Test Data Compression", Proc. Int. Conf. Electronics, Circuits and Systems, pp. 449-452, 2002.

[5] P. T. Gonciari, B. Al-Hashimi and N. Nicolici, "Improving compression ratio, area overhead, and test application time for system-on-a-chip test data compression/decompression", Proc. DATE Conf., pp. 604-611, 2002.

[6] I. Hamzaoglu and J. H. Patel, "Test set compaction algorithms for combinational circuits", Proc. Int. Conf. CAD, pp. 283-289, 1998.

[7] I. Hamzaoglu and J. H. Patel, "Reducing test application time for full scan embedded cores," Proc. IEEE Int. Symp. Fault Tolerant Computing, pp. 260-267, 1999.

[8] I. Hamzaoglu and J. H. Patel, " Reducing test application time for builtin-self-test test pattern generators", Proc. IEEE VLSI Test Symposium, pp. 369-375, 2000.

[9] A. Jas, C. V. Krishna and N. A. Touba, "Hybrid BIST based on weighted pseudo-random testing: a new test resource partitioning scheme," Proc. VLSI Test Symp., pp. 2-8, 2001.

[10] A. Khoche and J. Rivoir, "I/O bandwidth bottleneck for test: is it real?" Test Resource Partitioning Workshop, 2002.

[11] B. Koenemann et al., "A SmartBIST variant with guaranteed encoding," Proc. Asian Test Symp., pp. 325-330, 2001.

[12] K.-J. Lee, J.-J. Chen, C.-H. Huang, "Using a single input to support multiple scan chains," Proc. Int. Conf. CAD, pp. 74-78, 1998.

[13] $\mathrm{L}$. Li and $\mathrm{K}$. Chakrabarty, "Test data compression using dictionaries with fixed-length indices," Proc. VLSI Test Symp., pp. 219-224, 2003.

[14] J. Rajski et al., "Embedded deterministic test for low-cost manufacturing test," Proc. Int. Test Conf., pp. 301-310, 2002.

[15] H. Tang, S. M. Reddy and I. Pomeranz, "On reducing test data volume and test application time for multiple scan chain designs," Proc. Intl. Test Conf., pp. 1079-1088, 2003.

[16] M. Tehranipour, M. Nourani and K. Chakrabarty, "Nine-coded compression technique with application to reduced pin-count testing and flexible on-chip decompression," Proc. DATE Conf., pp. 1284-1289, 2004.

[17] H. Vranken et al., "ATPG padding and ATE vector repeat per port for reducing test data volume," Proc. Intl. Test Conf., pp. 1069-1076, 2003.

[18] F. G. Wolff and C. Papachristou, "Multiscan-based test compression and hardware decompression using LZ77", Proc. Int. Test Conf., pp. 331-339, 2002.

[19] A. Wurtenberger, C. S. Tautermann and S. Hellebrand, "A hybrid coding strategy for optimized test data compression", Proc. Int. Test Conf., pp. 451-459, 2003. 